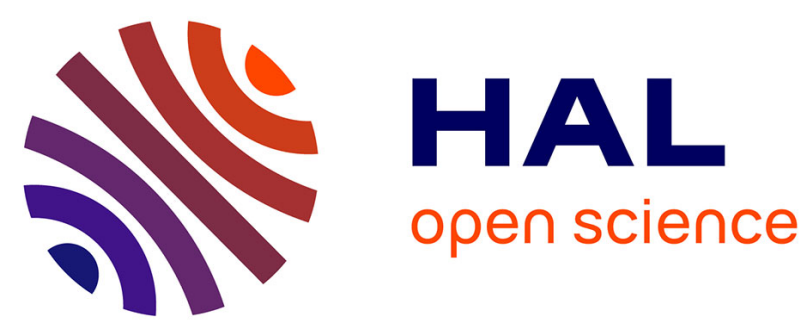

\title{
Measurement of guided mode wavenumbers in soft tissue-bone mimicking phantoms using ultrasonic axial transmission
}

Jiangang Chen, Josquin Foiret, Jean-Gabriel Minonzio, Maryline Talmant, Zhongqing Su, Li Cheng, Pascal Laugier

\section{To cite this version:}

Jiangang Chen, Josquin Foiret, Jean-Gabriel Minonzio, Maryline Talmant, Zhongqing Su, et al.. Measurement of guided mode wavenumbers in soft tissue-bone mimicking phantoms using ultrasonic axial transmission. Physics in Medicine and Biology, 2012, 57 (10), pp.3025 - 3037. 10.1088/00319155/57/10/3025 . hal-01394324

\section{HAL Id: hal-01394324 https://hal.sorbonne-universite.fr/hal-01394324}

Submitted on 9 Nov 2016

HAL is a multi-disciplinary open access archive for the deposit and dissemination of scientific research documents, whether they are published or not. The documents may come from teaching and research institutions in France or abroad, or from public or private research centers.
L'archive ouverte pluridisciplinaire HAL, est destinée au dépôt et à la diffusion de documents scientifiques de niveau recherche, publiés ou non, émanant des établissements d'enseignement et de recherche français ou étrangers, des laboratoires publics ou privés. 


\section{Measurement of guided mode wavenumbers in soft tissue-bone mimicking phantoms using ultrasonic axial transmission}

Jiangang Chen ${ }^{1}$, Josquin Foiret ${ }^{2,3}$, Jean-Gabriel Minonzio ${ }^{2,3}$, Maryline Talmant $^{2,3}$, Zhongqing Su ${ }^{1}$,

Li Cheng ${ }^{1}$ and Pascal Laugier ${ }^{2,3}$

${ }^{1}$ Department of Mechanical Engineering, The Hong Kong Polytechnic University, Kowloon, Hong Kong SAR

${ }^{2}$ UPMC (Univ Paris 6) Laboratoire d'Imagerie Paramétrique F-75005, Paris, France

${ }^{3}$ CNRS, UMR 7623, LIP, 15 rue de l'école de médecine F-75006, Paris, France

Email: jean-gabriel.minonzio@upmc.fr

short title: guided modes in soft tissue-bone mimicking phantoms

August $23^{\text {rd }} 2011$

Revised February $29^{\text {th }} 2012$

Submitted to: Physics in Medicine and Biology 


\begin{abstract}
Human soft tissue is an important factor that influences the assessment of human long bones using quantitative ultrasound (QUS) techniques. To investigate such influence, a series of soft tissue-bone phantoms (a bone mimicking plate coated with a layer of water, glycerol or silicon rubber) were ultrasonically investigated using a probe with multi-emitter and multi-receiver arrays in an axial transmission configuration. A singular value decomposition (SVD) signal processing technique was applied to extract the frequency-dependent wavenumbers of several guided modes. The results indicate that the presence of a soft tissue-mimicking layer introduces additional guided modes predicted by a fluid waveguide model. The modes propagating in the bone mimicking plate covered by the soft-tissue phantom are only slightly modified compared to their counterparts in the free bone-mimicking plate, and they are still predicted by an elastic transverse isotropic two-dimensional waveguide. Altogether these observations suggest that the soft tissue-bone phantoms can be modeled as two independent waveguides. Even in presence of the overlying soft tissue-mimicking layer, the modes propagating in the bonemimicking plate can still be extracted and identified. These results suggest that our approach can be applied for the purpose of the characterization of the material and structural properties of cortical bone.
\end{abstract}

PACS numbers

4320Ye Measurement methods and instrumentation,

$4380 \mathrm{Vj}$ Acoustical medical instrumentation and measurement techniques

$4320 \mathrm{Mv}$ Waveguides, wave propagation in tubes and ducts

4360Fg Acoustic array systems and processing, beam-forming

Key words: Axial transmission; Bone; Guided wave; Quantitative ultrasound; Soft tissue; Singular value decomposition (SVD); 


\section{Introduction}

The axial transmission technique, employing a set of emitter(s) and receiver(s) to measure long bones, like tibia and radius, is considered to be one of the most promising modalities among quantitative ultrasound (QUS) techniques. The shaft of long bones has been evidenced to act as a natural waveguide (Moilanen 2008, Talmant et al 2010). A number of studies have demonstrated experimentally the potential of this technique to assess bone mechanical and structural properties (Moilanen et al 2003a, Bossy et al 2004, Muller et al 2005). Several in vivo studies evidenced that the velocity of the first-arrival signal (FAS) allows discriminating osteoporotic from healthy patients (Moilanen et al 2003a, Knapp et al 2001, Barkmann et al 2000, Hans et al 1999, Talmant et al 2009, Kilappa et al 2011). In other studies, the signal processing techniques were adapted to identify several guided modes propagating in long bones, focusing on their velocity dispersion or attenuation properties. For example, the energetic late arrival (ELA), associated with the low frequency fundamental flexural $\mathrm{A}_{0}$-like plate guided mode, has been measured in vitro in human radius specimens (Moilanen et al 2006, Sasso et al 2009). Higher order guided modes have also been observed in vitro in bovine and human bone specimens (Lefebvre et al 2002, Lee and Yoon 2004, Le et al 2010, Protopappas et al 2006, Song et al 2011, Tatarinov et al 2011). In the above mentioned reports, the experiments were conducted in vitro on excised bones specimens, i.e., after removal of the overlying soft tissue.

While the feasibility of the identification and measurement of multiple guided modes has been demonstrated on long bones without overlying soft tissue on top of it, the influence of soft tissue on guided modes dispersion curves has not been extensively studied yet, apart from the studies reported by Moilanen et al (2008) dedicated to the fundamental flexural mode, i.e., $\mathrm{A}_{0}$ in a plate or $\mathrm{F}_{11}$ in a tube. Previous in vivo studies by these authors suggested that the measurement of a signal contribution consistent with the fundamental flexural mode was possible in vivo on the tibia in some patients (Moilanen et al 2003a, Moilanen et al 2007), while in other patients the identification of such a mode was difficult, presumably due to the presence of soft tissues on top of the bone. In a subsequent modeling 
study (Moilanen et al 2008), the authors reported an increasing mode density when the soft tissue thickness increases. From that latter study, the authors concluded that the identification of the flexural mode might be difficult when the soft tissue on top of the bone is thicker than a few millimetres. The presence of the soft tissue layer on top of long bones may be the cause for the ambiguity encountered in mode identification in vivo for some patients.

Our group has recently introduced a method for extracting guided mode phase velocities based on the singular value decomposition (SVD). This method consist in the analysis of the time responses for all possible pairs of emitter-receiver of a multi-emitter and multi-receiver array placed on top of the bone being investigated (Minonzio et al 2010, 2011a). High order guided modes are obtained from the maxima of the so-called Norm function in the temporal frequency - spatial frequency plane. The method has been shown to be efficient on bone mimicking plates made of a dissipative transverse isotropic elastic medium with characteristics mimicking those of actual bones (Minonzio et al 2011b).

The goal of the present work was to investigate the effect of overlying soft tissues on the propagation characteristics of guided modes in an axial transmission configuration by extending the method to the determination of the guided modes dispersion curves for a fluid-solid bilayer waveguide consisting of soft tissue-mimicking fluid or viscoelastic layers on top of a bone-mimicking plate. Our assumption is that the bilayer waveguide behaves as two uncoupled waveguides. Since the bonemimicking plate is a transverse isotropic solid and soft tissue-mimicking fluid is isotropic media, the problem in this study is described using a two dimensional model. The theory of guided mode propagation in plates is briefly summarized in section 2 , where the dispersion equations for a fluid plate and for a transverse isotropic elastic plate are reminded. The experimental procedure using a multielement transmitting and receiving transducer array is described in section 3, where we outline some specifics of the SVD-based signal processing technique. In section 4, the results of axial transmission experiments on a variety of bilayer waveguides designed to mimic the overlying soft tissues on top of cortical bone are displayed. The measured guided mode wavenumbers are compared to the theoretical 
predictions for the two uncoupled fluid and solid waveguides model. We conclude by a discussion of our results and a summary of our findings.

\section{Theoretical model}

In an axial transmission measurement configuration, a linear array of emitters and receivers is aligned along the bone axis $\left(O x_{1}\right.$ in figure 1$)$. In spite of the tubular shape of long bones, the propagation of guided waves in the cortical layer can be described in a first approximation by a plate model (Moilanen 2008, Talmant et al 2010). The plate model will thus be considered hereinafter. Consider a solid plate of thickness $h$ covered by a fluid layer of thickness $L$ (figure 1 ). The wavenumbers of the bilayer guided modes can be theoretically computed (Dayal and Kinra 1989, Yapura and Kinra 1995, Simonetti 2004). They depend on the thicknesses, the mass densities, the plate elastic coefficients $C_{i j}$ and the fluid longitudinal velocity $c_{f}$. However, for simplicity, following Simonetti (2004) and Wu and Yang (2011), the bilayer waveguide is assumed in a first approximation to behave mainly like two decoupled waveguides. It implies that a measured guided mode wavenumber $k^{e x p}$ corresponds to either a plate $k^{p}$ or a fluid wavenumber $k^{f}$. This approximation will be discussed in section 4.

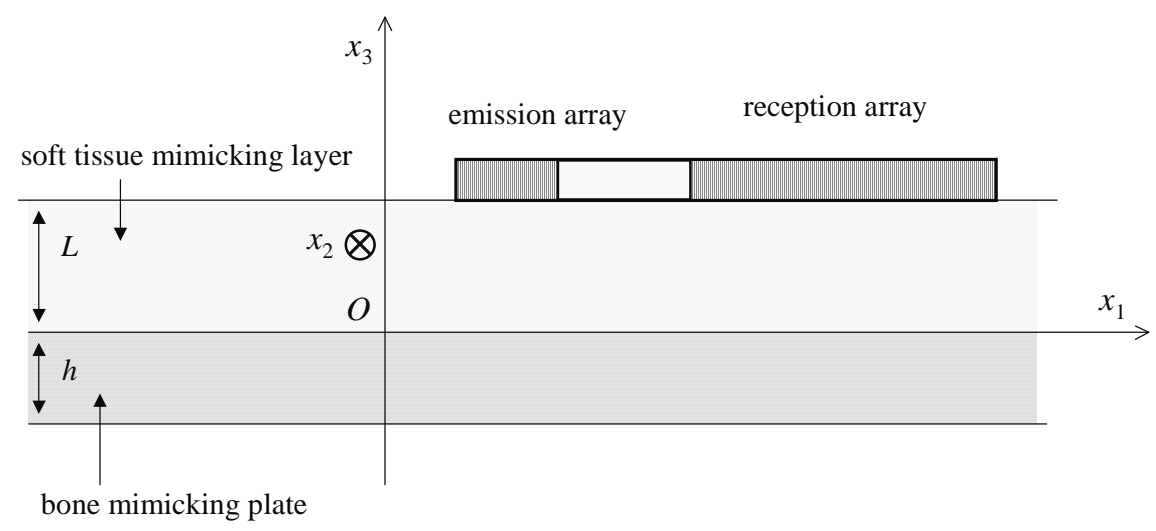

Figure 1. Geometry of the problem: an elastic plate, covered by a fluid layer, is inspected with a multiemitter and multi receiver array. 
First, consider a fluid layer of thickness $L$ with clamped-free boundary conditions (figure 1). The boundary conditions implies that the wavenumber $k_{3}$ along the $\left(O x_{3}\right)$ direction is equal to $(n+1 / 2) \pi L$, with $n$ an integer. Moreover, the wavenumber $k_{1}$ along the $\left(O x_{1}\right)$ direction, satisfies $k_{1}^{2}+k_{3}^{2}=\left(\omega / c_{f}\right)^{2}$, with $\omega$ the angular frequency and $c_{f}$ the longitudinal velocity in fluid. Thus the fluid-guided wavenumber, denoted $k^{f}$ and equal to $k_{1}$ satisfies (Royer and Dieulesaint 2000, Simonetti 2004)

$$
k^{f}=\left[\left(\frac{\omega}{c_{f}}\right)^{2}-\left(\frac{[n+1 / 2] \pi}{L}\right)^{2}\right]^{1 / 2},
$$

The different fluid guided modes are labeled according to their index $n$ equal to $0,1,2,3 \ldots$

Secondly, consider a free transverse isotropic elastic plate of thickness $h$ (figure 1). The direction $\left(O x_{1}\right)$ is the symmetry axis. Guided waves in the elastic plate are different from their counterparts in the fluid layer as the elastic plate supports the propagation of shear waves. Because the probe works in piston mode and does not excite horizontal shear displacement, only motion in the plane $\left(O x_{1} x_{3}\right)$ is considered. Thus a two dimensional approximation can be considered (Dayal and Kinra 1989, Rhee et al 2007). Following previous conditions, the relation between $k_{3}$ and $k_{1}$ satisfies (Dayal and Kinra 1989, Rhee et al 2007)

$$
k_{3 \pm}^{2}=\left(\frac{-M \pm \sqrt{M^{2}-4 N}}{2}\right)^{2} k_{1}^{2},
$$

where the sign \pm indicates the positive and negative $\left(O x_{3}\right)$ directions (figure 1). The terms $M$ and $N$ correspond to

$$
M=\frac{C_{11} C_{33}-C_{33} C_{55}-C_{13}{ }^{2}-\frac{\rho \omega^{2}}{k_{1}^{2}}\left(C_{33}+C_{55}\right)}{C_{33} C_{55}},
$$




$$
N=\frac{\left(\frac{\rho \omega^{2}}{k_{1}^{2}}-C_{11}\right)\left(\frac{\rho \omega^{2}}{k_{1}^{2}}-C_{55}\right)}{C_{33} C_{55}},
$$

where $\rho$ is the plate density, $\omega$ is the angular frequency, and $C_{11}, C_{33}, C_{13}$ and $C_{55}$ are the stiffness coefficients of the transverse isotropic plate. Following equation (32) of Rhee et al (2007), the dispersion equation of the symmetric Lamb modes $S_{n}$ can be written as

$$
\begin{aligned}
& \left(C_{33} R_{-} k_{3-}+C_{13} k_{1}\right)\left(R_{+} k_{1}+k_{3+}\right) \sin \left(k_{3+} h\right) \cos \left(k_{3-} h\right) \\
& -\left(C_{33} R_{+} k_{3+}+C_{13} k_{1}\right)\left(R_{-} k_{1}+k_{3-}\right) \sin \left(k_{3-} h\right) \cos \left(k_{3+} h\right)=0
\end{aligned},
$$

Following equation (34) of Rhee et al (2007), the dispersion equation for the anti-symmetric modes $\mathrm{A}_{n}$ is obtained by inverting the + and - subscripts inside the parentheses in (4a)

$$
\begin{aligned}
& \left(C_{33} R_{+} k_{3+}+C_{13} k_{1}\right)\left(R_{-} k_{1}+k_{3-}\right) \sin \left(k_{3+} h\right) \cos \left(k_{3-} h\right) \\
& -\left(C_{33} R_{-} k_{3-}+C_{13} k_{1}\right)\left(R_{+} k_{1}+k_{3+}\right) \sin \left(k_{3-} h\right) \cos \left(k_{3+} h\right)=0
\end{aligned},
$$

The terms $R_{+}$and $R_{-}$in (4) are given by

$$
R_{ \pm}=\frac{\left(\rho \omega^{2}-C_{11} k_{1}^{2}-C_{55} k_{3 \pm}^{2}\right)}{\left(C_{55}+C_{13}\right) k_{1} k_{3 \pm}}
$$

In the following, the theoretical wavenumbers $k_{f}$, associated with guided modes in the soft tissuemimicking layer, will be computed using (1). Likewise, the theoretical wavenumbers $k^{p}$, associated with the bone-mimicking plate, will be computed using (2) to (5), with $k^{p}$ equal to $k_{1}$. These theoretical wavenumbers will be compared with experimental data $k^{\text {exp }}$ in section 4 measured on a bone-mimicking plate covered by various soft tissue-mimicking layers. The experimental data are obtained using the experimental set up and the signal processing described in section 3 . 


\section{Material and Method}

\subsection{Experimental set up}

In order to acquire experimental data to compare with the model described in section 2, a custom made linear transducer array (Vermon, Tours, France) was used (figures 1 and 2). The characteristics of this probe have already been given in previous papers by our group (Minonzio et al 2010, 2011a). The probe contains $N^{\mathrm{E}}=5$ emitters and $N^{\mathrm{R}}=32$ receivers (pitch of the array: $0.8 \mathrm{~mm}$; minimum distance between both transmitting and receiving arrays: $11 \mathrm{~mm}$; central frequency $1 \mathrm{MHz}$; $-6 \mathrm{~dB}$ spectrum spanning the frequency range of 0.5 to $1.5 \mathrm{MHz}$ and the $-20 \mathrm{~dB}$ spectrum spanning the frequency range of 0.4 to $1.8 \mathrm{MHz}$ ). Such a configuration allowed 160 transmission-reception channels. During the measurement, each emitter was excited with wideband pulses. The radiofrequency signals recorded on the receivers were digitized ( 8 bits, $20 \mathrm{MHz}, 1024$ time samples) and finally stored on a computer for off-line analysis. Twenty radiofrequency signals were time averaged to increase signal-to-noise ratio. The driving electronics has been manufactured by Althaïs Technology (Tours, France).

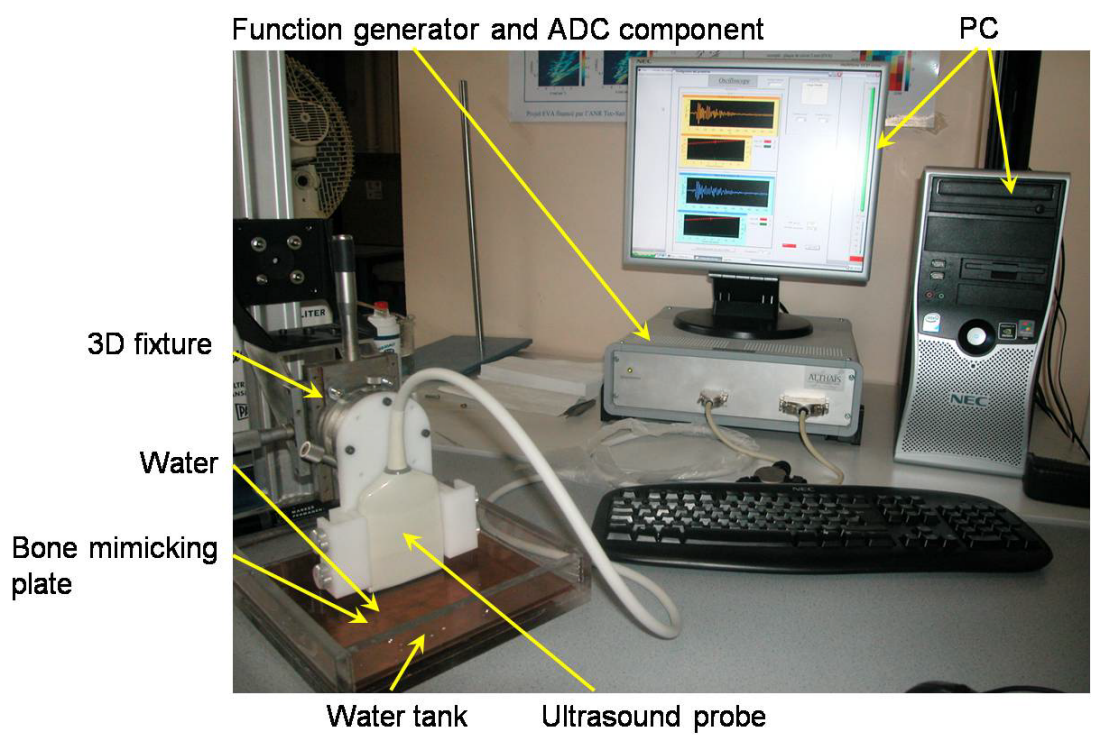

Figure 2. Experimental set-up 
A $2.3 \mathrm{~mm}$-thick bone-mimicking plate (Sawbones ${ }^{\circledR}$, Pacific Research Laboratories, Washington, USA) was used as a bone phantom. The thickness is typical of the cortical thickness of the human radius. The plate is made of oriented glass fibers embedded in epoxy. Its elastic properties, summarized in table 1 , are close to those of actual bones. The mass density $\rho$ of the bone mimicking plate is equal to $1.64 \mathrm{~g} . \mathrm{cm}^{-3}$. The stiffness coefficients $C_{11}, C_{33}, C_{44}$ and $C_{55}$ were deduced from bulk compression and shear velocities measured using contact transducers (Minonzio et al 2011b, Granke et al 2011). The stiffness coefficient $C_{13}$ was deduced from $C_{33}$ and $C_{44}$ using $C_{13}=C_{33}-2 C_{44}$.

Table 1. Elastic coefficients of the bone mimicking plate

$\begin{array}{lllll}\text { Elastic coefficients } & C_{11} & C_{33} & C_{55} & C_{13}\end{array}$

Numerical values $(\mathrm{GPa})$ 22.5 13.5 4.1 5.9

Three different soft tissue-mimicking layers have been used: water, $98 \%$ glycerol (VWR International, Fontenay-sous-Bois, France) and silicon rubber. The silicon rubber was artificially produced by mixing three substances, i.e., 4600A, 4600B and AK35 (all from Wacker Chemicals Ltd., Béziers, France) with the proportions of $1: 0.1: 1.6$. The longitudinal velocity $c_{f}$, density $\rho$ and attenuation $\alpha$ of the three soft tissue phantoms are given in table 2 . The longitudinal velocity in the silicon has been measured using two contact transducers. These materials, easy to handle, were chosen, owing to the similarity of their acoustical properties with those of soft tissues (table 2). Water is similar to soft tissues in term of velocity. Silicon rubber, $98 \%$ glycerol and soft tissue have similar attenuation values. The longitudinal velocities in the silicon rubber and $98 \%$ glycerol are respectively lower and higher than that in soft tissues. These material have been used in order to test the model with a wide range of longitudinal velocity and attenuation values. Moreover, considering that soft tissues are not pure fluid but 
sustains shear stresses (Moilanen et al 2003b), silicon rubber is used as a tissue equivalent material (Njeh et al 1999).

The bone phantom was placed into a tank, and supported by four tiny cylinders (height: $2 \mathrm{~mm}$ ). First, reference measurements were taken with the bone-mimicking phantom measured without any soft tissue-mimicking layer on top of it, with the probe being simply coupled to the bone plate with an acoustically transparent gel (Aquasonic ${ }^{\circledR}$, Parker Laboratories, Inc., NJ, USA). Subsequently, the fluid (water or $98 \%$ glycerol) was introduced into the tank. The position and the parallelism of the probe with respect to the plate could be adjusted by tuning the translational and rotational stages (figure 2). The position of the probe was adjusted above the plate to leave a $4 \mathrm{~mm}$-thick and then $8 \mathrm{~mm}$-thick layer of water, and subsequently a 4 mm-thick layer of $98 \%$ glycerol. Following the measurements with water and 98\% glycerol, a $4 \mathrm{~mm}$-thick layer of silicon rubber was placed on top of the bone-mimicking plate, such that any air bubbles in between could be carefully eliminated. The gel was used to improve the coupling between the probe and the silicon rubber. 
Table 2. Longitudinal velocity $c_{f}$, density $\rho$ and attenuation $\alpha$ of the different soft tissue-mimicking materials considered in the experiments.

\begin{tabular}{|c|c|c|c|c|}
\hline material & $c_{f}\left(\mathrm{~ms}^{-1}\right)$ & $\rho\left(\mathrm{gcm}^{-1}\right)$ & $\alpha\left(\mathrm{dB} \cdot \mathrm{cm}^{-1}\right)$ at $1 \mathrm{MHz}$ & References \\
\hline Silicon rubber & 1230 & 0.8 & 1 & $\begin{array}{l}\text { (Itsumi et al 2009, } \\
\text { Zheng et al 2011) }\end{array}$ \\
\hline Water & 1480 & 1.0 & $10^{-2}$ & $\begin{array}{l}\text { (Tang et al 1988, } \\
\text { Culjat et al 2010) }\end{array}$ \\
\hline Soft tissues & $1430-1600$ & $0.95-1.05$ & $0.5-1$ & $\begin{array}{l}\text { (Culjat et al 2010, } \\
\text { Mast 2000) }\end{array}$ \\
\hline Glycerol (98\%) & 1910 & 1.26 & 0.2 & $\begin{array}{c}\text { (Bossy et al 2004, } \\
\text { Fergusson et al 1954, } \\
\text { Tang et al 1988, } \\
\text { Figueiredo et al 2009) }\end{array}$ \\
\hline
\end{tabular}

\subsection{Signal processing}

The SVD-based signal processing technique has been described in detail elsewherere (Minonzio et al 2010, 2011a). Only the main steps are recalled here. The recorded radiofrequency signals $r_{i j}(t)$, with $i$ and $j$ the emission and reception indices ranging from 1 to $N^{\mathrm{E}}$ and 1 to $N^{\mathrm{R}}$, respectively, are first Fouriertransformed. The singular value decomposition is subsequently applied to the $N^{\mathrm{E}} \times N^{\mathrm{R}}$ sub-matrices, i.e., the matrices $R_{i j}(f)$ at each frequency. The signal subspace is determined by applying a first threshold $t_{1}$ on the singular values. The singular values below that threshold are considered associated with the noise 
subspace. The $M$ singular values above this threshold are associated with the signal subspace and their associated reception singular vectors, denoted $\mathbf{U}_{n}$, are a basis of the experimental guided modes. The threshold $t_{1}$ is heuristically selected. Then, the so-called Norm function is defined by the following equation

$$
\operatorname{Norm}(f, k)=\sum_{n=1}^{M}\left|\left\langle\mathbf{U}_{n} \mid \mathbf{e}^{p w}(k)\right\rangle\right|^{2},
$$

where $\mathbf{e}^{p w}(k)$ corresponds to a spatial plane wave at the spatial frequency $k$ defined on the reception array. Its norm is defined equal to 1 .

The scalar product $\left\langle\mathbf{U}_{n}\right| \mathbf{e}^{p w}(k)>$ corresponds to the normalized spatial Fourier transform of the singular vector $\mathbf{U}_{n}$. Thus, the SVD can be interpreted as a denoising step between the temporal and spatial Fourier transforms. If $k$ corresponds to a guided mode wavenumber $k_{\text {exp }}$, the Norm function is close to 1 , and the guided mode is projected in the singular vectors basis. If $k$ does not correspond to a guided mode wavenumber, the Norm function is small compared to 1 . Subsequently, a second heuristically chosen threshold $t_{2}$ is applied to the Norm function, the values of which larger than this threshold correspond to the guided modes wavenumbers $k_{\text {exp }}$.

As an example, the case of the $2.3 \mathrm{~mm}$-thick bone-mimicking plate alone (i.e., without fluid layer on top) in discussed. First, the corresponding Norm function given by (6) is shown in figure 3(a) in the $(f, k)$ plane. The retained maxima correspond to the experimental wavenumbers $k^{e x p}$ are marked with crosses in figure 3(b). They are compared with the theoretical plate modes $k^{p}$ computed from (2) to (5), using the stiffness coefficients given in table 1. The modes are labeled $\mathrm{S}_{n}$ or $\mathrm{A}_{n}$ according to their symmetry or anti-symmetry nature and their cutoff frequency $\times$ thickness product (denoted $f_{c} . h$ ). Three modes with transverse asymptotic behavior are identified: $\mathrm{A}_{1}\left(f_{c} \cdot h=c_{\mathrm{T}}^{\perp} / 2\right), \mathrm{S}_{2}\left(c_{\mathrm{T}}^{\perp}\right), \mathrm{S}_{4}\left(2 c_{\mathrm{T}}{ }^{\perp}\right)$, where $c_{\mathrm{T}}$ denotes the shear wave velocity, and the superscript ${ }^{\perp}$ the direction perpendicular to the fibers (Minonzio et al 2011b). Moreover, one mode $\left(\mathrm{S}_{1}\right)$ with longitudinal asymptotic behavior is identified $\left(f_{c} \cdot h=c_{\mathrm{L}}{ }^{\perp} / 2\right)$, 
with $c_{\mathrm{L}}{ }^{\perp}$ being the compression wave velocity in the direction perpendicular to the fibers. The $\mathrm{S}_{0}$ mode is also measured at low frequencies. The experimentally derived guided modes wavenumbers $k_{\exp }(f)$ are overall in good agreement with the theoretical Lamb modes wavenumbers.

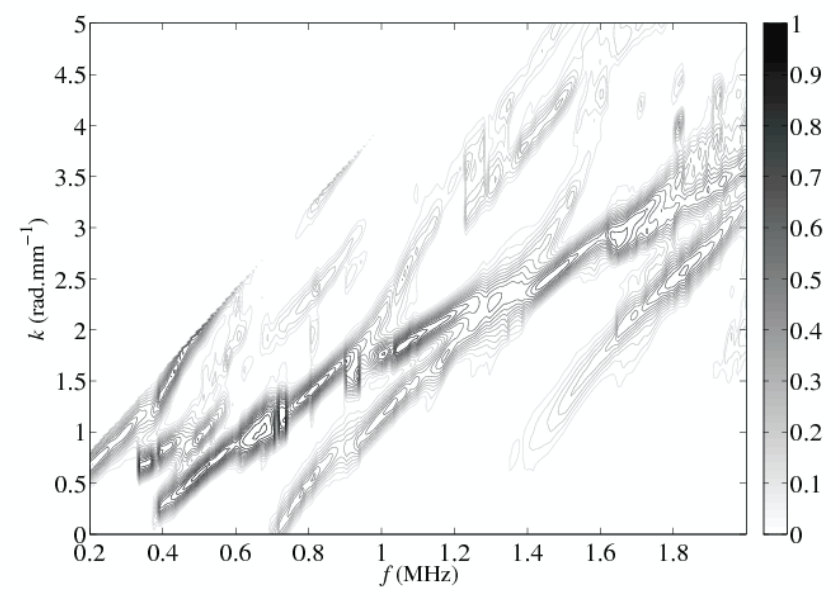

(a)

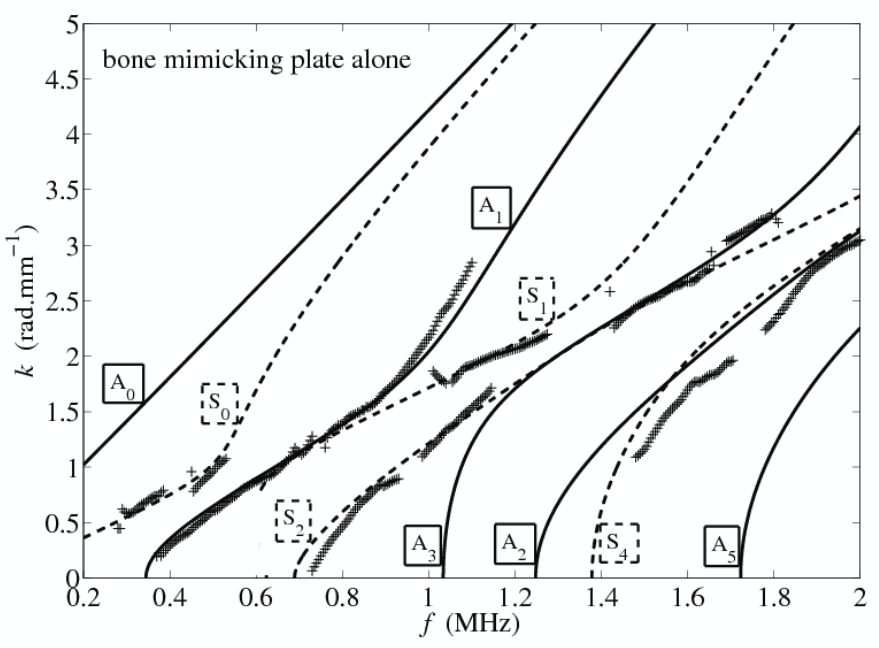

(b)

Figure 3. Norm function from the $2.3 \mathrm{~mm}$-thick bone-mimicking plate alone (a) and corresponding experimental guided mode wavenumbers $k^{e x p}$ (black crosses), obtained from the Norm function maxima, compared with theoretical plate modes $k^{p}$, shown with black continuous (anti-symmetric $\mathrm{A}_{n}$ ) and dashed (symmetric $\mathrm{S}_{n}$ ) lines (b). 


\section{Experimental results and discussion}

With the afore-addressed model and method, the coupling effect of soft tissue was interrogated by introducing four different soft tissue mimicking layers over the bone mimicking plate: water (layer thickness: 4 and $8 \mathrm{~mm}$ ), $98 \%$ glycerol (layer thickness: $4 \mathrm{~mm}$ ) and silicon rubber (layer thickness: 4 $\mathrm{mm})$. The results obtained with the four soft tissue-mimicking layers on top of the bone-mimicking bone plate are shown in figure 4 . The experimental wavenumbers $k^{e x p}$ are plotted against frequency together with the theoretical guided modes wavenumbers of the free bone-mimicking plate $k^{p}$ (already presented in figure 3) and of the fluid layers. The wavenumbers of the theoretical guided modes in the free fluid layers, denoted $k^{f}$, are computed using (1) and the fluid velocity values given in table 2 . The fluid modes are labeled with integers, denoted $n$, starting from 0 .

A visual examination of the results shown in panels a and $b$ of figure 4 reveals that the fluid-solid bilayer supports the propagation of additional modes compared to the bone mimicking plate alone (figure 3). There is an overall agreement between these new modes and the theoretical predictions from the fluid layer model, even in the case of viscoelastic soft tissue-mimicking media ( $98 \%$ glycerol or silicon). Note also that the modes of the silicon layer, which behaves more like a soft viscoelastic solid than a fluid, are still predicted by the fluid model. In addition, such an agreement is evaluated using

$$
\text { error }=\sqrt{\frac{\sum_{f}\left\|k_{n}^{\exp }-k_{n}^{\text {th }}\right\|^{2}}{\sum_{f}\left\|k_{n}^{\exp }\right\|^{2}}},
$$

where $k_{n}^{\text {th }}$ corresponds to $n^{\text {th }}$ theoretical fluid $\left(k_{f}\right)$ or plate $\left(k_{p}\right)$ mode. For the plate modes, the errors are equivalent with (figure 4) or without (figure 3 ) the fluid layer. The error ranges from $4 \%\left(A_{1}\right.$ and $\left.S_{1}\right)$ to $9 \%\left(\mathrm{~S}_{0}, \mathrm{~S}_{2}\right.$ and $\left.\mathrm{S}_{4}\right)$. For the fluid modes, the error varies from $2 \%$ ( $n=4$ in the $4 \mathrm{~mm}$ water case) to $20 \%$ ( $n=7$ in the $4 \mathrm{~mm}$ glycerol case). The average of the fluid mode error for the four cases is equal to $5 \%$.

The number of observed fluid-guided waves increases with the layer thickness $L$ as predicted by the model. For instance, more modes are observed for the $8 \mathrm{~mm}$-thick water layer (panel $\mathrm{b}$ ), with $n$ 
ranging from 3 to 17, than for the 4 mm-thick water layer (panel a), with $n$ ranging from 0 to 6 . The slopes of the dispersion curves of the fluid guided modes tend towards $\omega / c_{f}$ as the frequency increases [equation (1)]. Thus considering the 4 mm-thick fluid layers only, the slope of the dispersion curves at high frequencies are steeper for the silicon (panel d), compared to water (panel a) and 98\% glycerol (panel c) in agreement with the values given in table 2. 


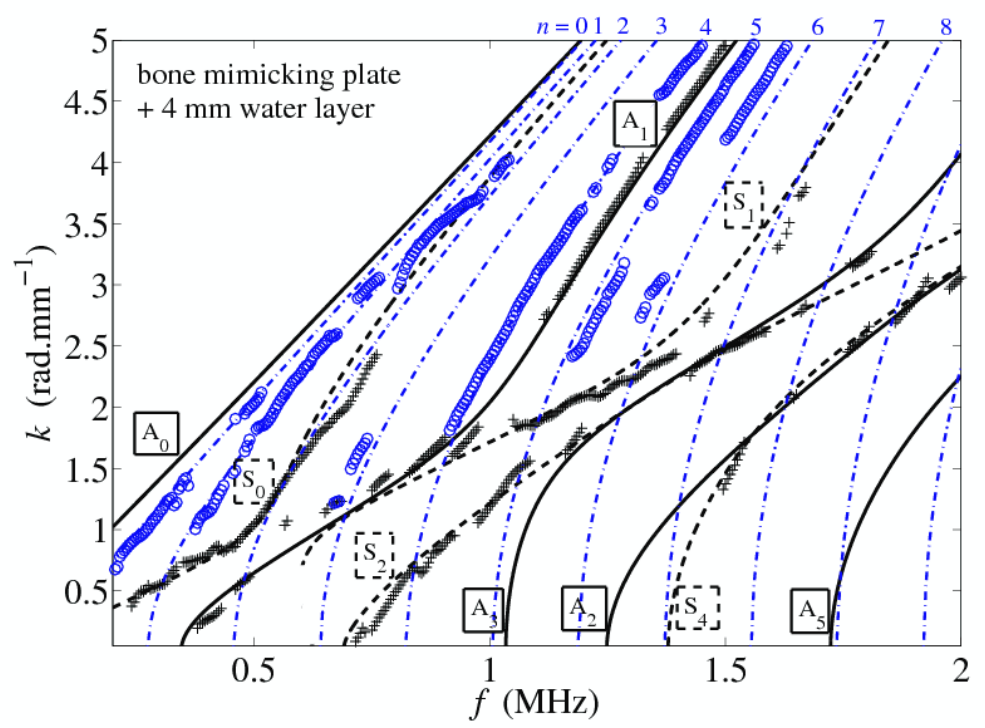

(a)

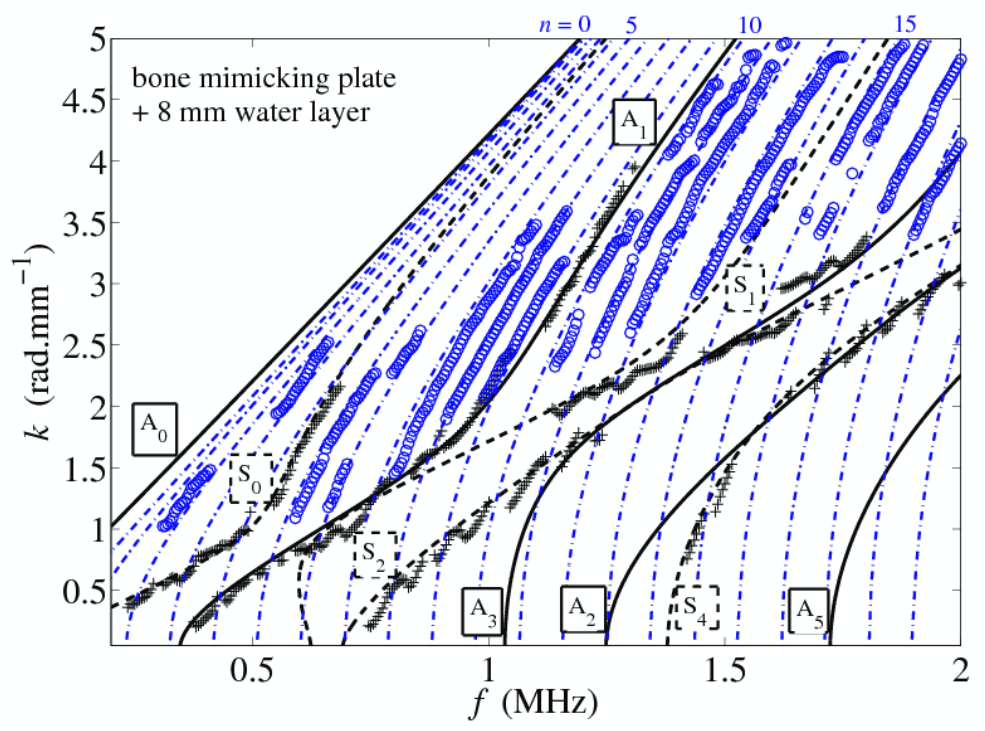

(b) 


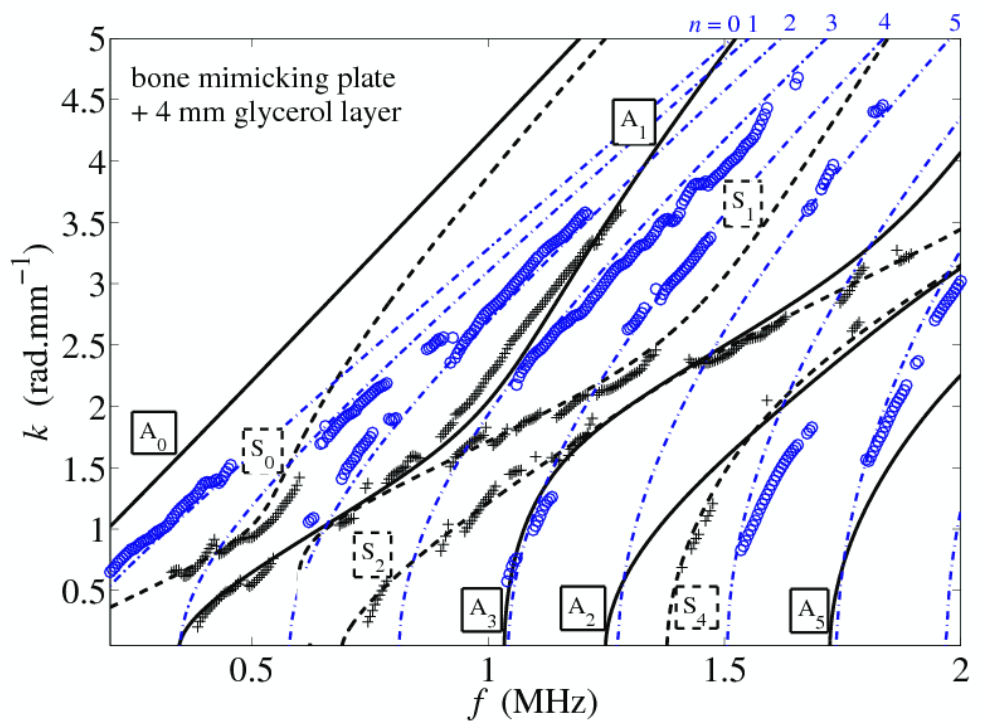

(c)

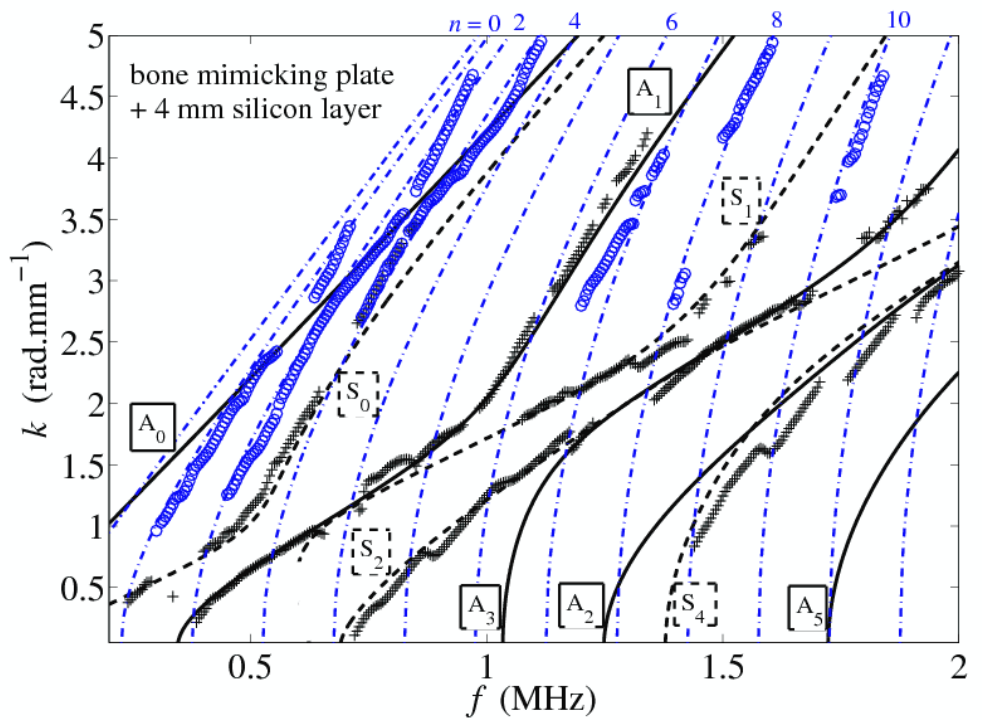

(d)

Figure 4. (color online) Experimental guided mode wavenumbers $k^{e x p}$ (blue circles and black crosses) obtained from the bone-mimicking plate in the presence of a soft tissue-mimicking layer: $4 \mathrm{~mm}$ thick water layer (a); 8 mm-thick water layer (b); 4 mm-thick 98\% glycerol layer (c); 4 mm-thick silicon rubber (d). The experimental wavenumbers are compared with the theoretical plate Lamb modes $k^{p}$, shown with black continuous (anti-symmetric $\mathrm{A}_{n}$ ) and dashed (symmetric $\mathrm{S}_{n}$ ) lines and theoretical fluid layer modes $k^{f}$, shown with blue dashed-dot lines (labeled with index $n$ ). The experimental wavenumbers are shown with symbols: black crosses are associated with plate modes, blue circles are associated with fluid layer modes. 
These additional modes are marked with circles on figure 4, whereas the experimental wavenumbers associated with the bone-mimicking plate modes are marked with crosses. The criteria for resolving the question of whether an observed trace is the result of a mode propagating in the soft tissuemimicking layer or in the bone-mimicking plate are the distances between the experimental trace and the simulated traces: an experimental trace is associated to the closest theoretical dispersion curve. In the present study, mode selection remains purely manual. For most of the cases, both the fluid and bonemimicking plate modes do not overlap so that their differentiation is trivial. When two modes overlap, the proper mode identification remains ambiguous, as is the case for mode $S_{0}$ which overlaps with the water layer mode $n=2$ (panel a) and $n=4$ (panel b) or for the mode $\mathrm{A}_{3}$ which overlaps with the $98 \%$ glycerol layer mode $n=4$ (panel c).

While most of the modes associated with the bone-mimicking plate with the soft tissuemimicking layer on top of it (figure 4, panels a-d) remain almost unchanged compared to their counterparts observed on the free bone-mimicking plate (figure 3), and are still predicted by an elastic transverse isotropic waveguide, we note slight differences like discontinuities in the trace of mode $A_{1}$ (panels a and c) or scalloping in the traces of mode $S_{1}$ and $S_{2}$ (panel b), presumably due to interferences with the fluid guided modes. However, these differences remain small, and should not prevent mode identification for the purpose of bone characterization.

We proposed to model the fluid-solid bilayer assemblies that were specifically designed to mimic the soft tissue layer on top of long bones as two uncoupled fluid and solid waveguides, each of which supporting its own guided modes. Results from theoretical computations based on this hypothesis appear to be consistent with measurements of both fluid and solid guided modes depending upon specific details of the soft-tissue mimicking layer considered.

These observations suggest that the modes propagating in the bone-mimicking plate can be extracted and identified in presence of the soft tissue-mimicking layer. Toward this goal, one needs to 
properly model the frequency-dependent wavenumbers of the soft tissue layer, which should be feasible once the thickness of the soft tissue has been estimated, from simple pulse echo measurements for example. Note that the speed of sound in the soft tissue is also required for the model. But because the speed of sound in soft biological tissues is fairly constant, its value derived from literature should be sufficient. It is then conceivable that meaningful bone properties can be recovered by carrying out an inversion scheme comparing the identified cortical bone modes to a theoretical plate model.

There are limitations to the preliminary work described in this brief report. The main limitation, of course, is that the measurements are performed in phantoms instead of actual bones. The phantoms only approximate the structure and acoustical properties of soft tissue and cortical bone. Additional complexities will arise from the inhomogeneity of real soft tissues and bones. It is not clear yet whether in vivo application of this approach will be feasible, until a range of experimental data has been analyzed. An advantage of the present study is that a custom made probe designed for clinical examinations (Talmant et al 2009) has been used to conduct experiments on the phantoms. On the other hand, a plate model has been used in the study, while long bones are tubular-shaped. However, previous studies of axial transmission in cortical bone have previously demonstrated a high level of consistency of the plate model with experimental data (Moilanen et al 2006).

\section{Conclusion}

In summary, this study of the coupling effect of soft tissue-mimicking layers on top of a bonemimicking plate on guided wave propagation suggests that the presence of a soft tissue-mimicking layer introduces additional guided modes, but that both layers can be considered as independent waveguides. These results indicate that the modes propagating in the bone-mimicking plate can still be identified using an elastic transverse isotropic two-dimensional waveguide model and that they could be used for the purpose of the characterization of the material and structural properties of the bone waveguide. Future studies will be extended to in vivo measurements. 


\section{Acknowledgements:}

This work has been supported by ANR project "COSTUM" 09-TECS-005-03 (2009-2012).

\section{References}

Barkmann R, Kantorovich E, Singal C, Hans D, Genant H K, Heller M and Gluer C C 2000 A new method for quantitative ultrasound measurements at multiple skeletal sites - First Results of precision and fracture discrimination J. Clin. Densitom. 3 1-7

Bossy E, Talmant M, Defontaine M, Patat F and Laugier P 2004 Bidirectional axial transmission can improve accuracy and precision of ultrasonic velocity measurement in cortical bone: A validation on test materials IEEE Trans. Ultrason. Ferroelectr. Freq. Control 51 71-9

Culjat M O, Goldenberg D, Tewari P and Singh R S 2010 A review of tissue substitutes for ultrasound imaging Ultrasound. Med. Biol. 36 861-73

Dayal V and Kinra V K 1989 Leaky Lamb Waves in an Anisotropic Plate .1. an Exact Solution and Experiments J. Acoust. Soc. Am $\mathbf{8 5} 2268-76$

Fergusson F A A, Guptill E W and MacDonald A D 1954 Velocity of sound in glycerol J. Acoust. Soc. Am. 26 67-9

Figueiredo M K K, Costa-Felix R P B, Alvarenga A V, Maggi L E, Portilho M F, Souza M N and Romeiro G A 2009 Study, Development, and Implementation of Analysis Technique of Biphasic Attenuation Systems Using Ultrasound. In: Xix Imeko World Congress: Fundamental and Applied Metrology, Proceedings, (Budapest: Imeko) pp 2634-7

Granke M, Grimal Q, Saïed A, Nauleau P, Peyrin F and Laugier P 2011 Change in porosity is the major determinant of the variation of cortical bone elasticity at the millimeter scale in aged women Bone 49, 1020-6

Hans D, Srivastav S K, Singal C, Barkmann R, Njeh C F, Kantorovich E, Gluer C C and Genant H K 1999 Does combining the results from multiple bone sites measured by a new quantitative ultrasound device improve discrimination of hip fracture? J. Bone Miner. Res. 14 644-51

Itsumi K, Hosono Y, Yamamoto N and Yamashita Y J 2009 Low acoustic attenuation silicone rubber lens for medical ultrasonic array probe IEEE Trans. Ultrason. Ferroelectr. Freq. Control 56 870-4

Kilappa V, Moilanen P, Xu L, Nicholson P H F, Timonen J and Cheng S 2011 Low-frequency axial ultrasound velocity correlates with bone mineral density and cortical thickness in the radius and tibia in pre- and postmenopausal women Osteoporosis Int. 22 1103-13

Knapp K M, Blake G M, Spector T D and Fogelman I 2001 Multisite quantitative ultrasound: Precision, age- and menopause-related changes, fracture discrimination, and T-score equivalence with dualenergy X-ray absorptiometry Osteoporosis Int. 12 456-64

Le L H, Gu Y J, Li Y P and Zhang C 2010 Probing long bones with ultrasonic body waves Appl. Phys. Lett. 96114102

Lee K I and Yoon S W 2004 Feasibility of bone assessment with leaky Lamb waves, in bone phantoms and a bovine tibia J. Acoust. Soc. Am. 115 3210-7

Lefebvre F, Deblock Y, Campistron P, Ahite D and Fabre J J 2002 Development of a new ultrasonic technique for bone and biomaterials in vitro characterization J. Biomed. Mater. Res. 63 441-6 
Mast T D 2000 Empirical relationships between acoustic parameters in human soft tissues Acoustics Research Letters Online 137-42

Minonzio J G, Talmant M and Laugier P 2010 Guided wave phase velocity measurement using multiemitter and multi-receiver arrays in the axial transmission configuration J. Acoust. Soc. Am 127 2913-9

Minonzio J G, Talmant M and Laugier P 2011a Measurement of guided mode wave vectors by analysis of the transfer matrix obtained with multi-emitters and multi-receivers in contact Journal of Physics: Conference Series 269012003

Minonzio J G, Foiret J, Talmant M and Laugier P 2011b Impact of attenuation on guided mode wavenumber measurement in axial transmission on bone mimicking plates J. Acoust. Soc. Am. 130, 3574-82

Moilanen P 2008 Ultrasonic guided waves in bone IEEE Trans. Ultrason. Ferroelectr. Freq. Control 55 1277-86

Moilanen P, Nicholson P H F, Kärkkäinen T, Wang Q, Timonen J and Cheng S 2003a Assessment of the tibia using ultrasonic guided waves in pubertal girls Osteoporosis Int 14 1020-7

Moilanen P, Nicholson P H F, Kilappa V, Wang Q, Timone J and Cheng S 2003b The role of soft tissue in ultrasonic guided wave measurement in bone. In: 5th World Congress on Ultrasonics (WCU), (Paris, France) pp 899-902

Moilanen P, Nicholson P H F, Kilappa V, Cheng S and Timonen J 2006 Measuring guided waves in long bones: Modeling and experiments in free and immersed plates Ultrasound. Med. Biol. 32 709-19

Moilanen P, Talmant M, Bousson V, Nicholson P H F, Cheng S, Timonen J and Laugier P 2007 Ultrasonically determined thickness of long cortical bones: Two-dimensional simulations of in vitro experiments J. Acoust. Soc. Am. 122 1818-26

Moilanen P, Talmant M, Kilappa V, Nicholson P, Cheng S L, Timonen J and Laugier P 2008 Modeling the impact of soft tissue on axial transmission measurements of ultrasonic guided waves in human radius J. Acoust. Soc. Am. 124 2364-73

Muller M, Moilanen P, Bossy E, Nicholson P, Kilappa V, Timonen J, Talmant M, Cheng S and Laugier P 2005 Comparison of three ultrasonic axial transmission methods for bone assessment Ultrasound. Med. Biol. 31 633-42

Njeh C F, Kearton J R, Hans D and Boivin C M 1999 The use of quantitative ultrasound to monitor fracture healing: A feasibility study using phantoms Med. Eng. Phys. 20 781-6

Protopappas V C, Fotiadis D I and Malizos K N 2006 Guided ultrasound wave propagation in intact and healing long bones Ultrasound. Med. Biol. 32 693-708

Rhee S H, Lee J K and Lee J J 2007 The group velocity variation of Lamb wave in fiber reinforced composite plate Ultrasonics 47 55-63

Royer D and Dieulesaint E 2000 Elastic Waves in Solids I (New York, NY: Springer)

Sasso M, Talmant M, Haiat G, Naili S and Laugier P 2009 Analysis of the Most Energetic Late Arrival in Axially Transmitted Signals in Cortical Bone IEEE Trans. Ultrason. Ferroelectr. Freq. Control 56 2463-70

Simonetti F 2004 Lamb wave propagation in elastic plates coated with viscoelastic materials J. Acoust. Soc. Am. 115 2041-53

Song X, Ta D and Wang W 2011 Analysis of superimposed ultrasonic guided waves in long bones by the joint approximative diagonialization of eigen-matrices algorithm Ultrasound Med. Biol. 37 170413

Talmant M, Foiret J and Minonzio J G 2010 Bone Quantitative ultrasound Chap. 7 Guided waves in cortical bones (New York: Springer) pp. 147-79

Talmant M, Kolta S, Roux C, Haguenauer D, Vedel I, Cassou B, Bossy E and Laugier P 2009 In vivo performance evaluation of bi-directional ultrasonic axial transmission for cortical bone assessment Ultrasound. Med. Biol. 35 912-9

Tang X, Toksöz M, Tarif P and Wilkens R 1988 A method for measuring acoustic wave attenuation in the laboratory J. Acoust. Soc. Am. 83 453-62 
Tatarinov A, Sarvazyan A, Beller G and Felsenberg D 2011 Comparative Examination of Human Proximal Tibiae In Vitro by Ultrasonic Guided Waves and pQCT Ultrasound Med. Biol. 201137 1791-1801

Yapura C L and Kinra V K 1995 Guided-Waves in a Fluid Solid Bilayer Wave Motion 21 35-46

Wu C H and Yang C H 2011 Guided waves propagating in a bi-layer system consisting of a piezoelectric plate and a dielectric fluid layer IEEE Trans. Ultrason. Ferroelectr. Freq. Control 58 1612-8

Zheng Y P, Chen J and Ling H Y 2011 Development of an Ultrasound Platform for the Evaluation of Plantar Soft Tissue Properties: A Feasibility Study on Silicone Phantom Feet Instrum. Sci. Technol. 39 248-60 